University of South Carolina

Scholar Commons

7-2005

\title{
Calibration and Evaluation of an Objective Measure of Physical Activity in Preschool Children
}

John R. Sirard

Stewart G. Trost

Karin A. Pfeiffer

Marsha Dowda

University of South Carolina - Columbia, mdowda@mailbox.sc.edu

Russell R. Pate

University of South Carolina - Columbia, rpate@mailbox.sc.edu

Follow this and additional works at: https://scholarcommons.sc.edu/

sph_physical_activity_public_health_facpub

Part of the Public Health Commons

\section{Publication Info}

Published in Journal of Physical Activity and Health, Volume 2, Issue 3, 2005, pages 345-357.

Sirard, J. R., Trost, S. G., Pfeiffer, K. A., Dowda, M., \& Pate, R. R. (2005). Calibration and evaluation of an objective measure of physical activity in prechool children. Journal of Physical Activity and Health, 2(3),345-357.

(c) Journal of Physical Activity and Health, 2005, Human Kinetics

This Article is brought to you by the Physical Activity and Public Health at Scholar Commons. It has been accepted for inclusion in Faculty Publications by an authorized administrator of Scholar Commons. For more information, please contact digres@mailbox.sc.edu. 


\title{
Calibration and Evaluation of an Objective Measure of Physical Activity in Preschool Children
}

\begin{abstract}
John R. Sirard, Stewart G. Trost, Karin A. Pfeiffer, Marsha Dowda, and Russell R. Pate

Background: The purposes of this study were 1) to establish accelerometer count cutoffs to categorize activity intensity of 3 to 5 -y old-children and 2) to evaluate the accelerometer as a measure of children's physical activity in preschool settings. Methods: While wearing an ActiGraph accelerometer, 16 preschool children performed five, 3-min structured activities. Receiver Operating Characteristic (ROC) curve analyses identified count cutoffs for four physical activity intensities. In 9 preschools, 281 children wore an ActiGraph during observations performed by three trained observers (interobserver reliability $=0.91$ to 0.98 ). Results: Separate count cutoffs for 3, 4, and 5-y olds were established. Sensitivity and specificity for the count cutoffs ranged from $86.7 \%$ to $100.0 \%$ and $66.7 \%$ to $100.0 \%$, respectively. ActiGraph counts $/ 15 \mathrm{~s}$ were different among all activities $(P<0.05)$ except the two sitting activities. Correlations between observed and ActiGraph intensity categorizations at the preschools ranged from 0.46 to $0.70(P<0.001)$. Conclusions: The ActiGraph count cutoffs established and validated in this study can be used to objectively categorize the time that preschool-age children spend in different physical activity intensity levels.
\end{abstract}

Key Words: accelerometer, measurement, young children, validity

Childhood obesity, in the US and many other countries, has increased dramatically in the past decade, ${ }^{1-3}$ and it is likely that decreased physical activity and increased sedentary behaviors are significantly related to this trend. ${ }^{4.5}$ Although increases in the prevalence of overweight and obesity are evident in children as young as 3 to $5 \mathrm{y},{ }^{1}$ little is known about the physical activity and sedentary behaviors of these preschool-age children. In addition, the long-term health effects of physical activity and sedentary behavior in very young children are not well understood. To clarify the effects of physical activity on overweight and obesity in preschool children, and to identify the relationship between physical activity and other health parameters

Sirard, Pfeiffer, Dowda, and Pate are with the Dept of Exercise Science, University of South Carolina, Columbia, SC 29208; Sirard is also with the Stanford Prevention Research Center, Stanford University, Stanford, CA 94305. Trost is with the Dept of Kinesiology and Community Health Institute, Kansas State University, Manhattan, KS 66506. 
in children of this age, a valid and reliable measure is needed that can detect the frequency, intensity, and duration of young children's physical activity.

An expert panel organized by the Centers for Disease Control and Prevention identified the need for a valid measure of physical activity in young children as a research priority. ${ }^{6}$ Most of the physical activity measures widely used to date have been problematic in this age group. Self-report measures are not recommended for children under age $10,{ }^{7}$ and teacher or parent proxy measures have not performed well compared to objectively measured physical activity. ${ }^{8-10}$ Direct observation can provide a valid assessment of children's physical activity but is costly and timeconsuming, ${ }^{11}$ making it impractical for large-scale epidemiological research. While there might be some level of "Hawthorne" effect or reactivity from using direct observation, Puhl et al. reported that less than $17 \%$ of the 5 and 6 y olds observed in their study reacted to the observers. ${ }^{12}$

Accelerometers provide an objective measure of physical activity and can be used in a wide range of settings and with people of virtually all ages. To date, however, they have been used only rarely to assess the physical activity levels of preschool-age children. ${ }^{13-16}$ Although previous studies have found positive associations between total activity determined by accelerometry and directly observed activity, ${ }^{13-16}$ fewer studies have explored an accelerometer's ability to categorize preschool children's activity by intensity (i.e., sedentary, light, moderate, vigorous). Identifying the amount of time spent in the various intensity categories will provide a better understanding of young children's daily patterns of activity and can provide target activity levels for future physical activity intervention programs. The primary purpose of this study was to calibrate a uniaxial accelerometer in preschool children by establishing count cutoffs that could be used to categorize activity intensity. A secondary purpose was to evaluate the validity of the count cutoffs using direct observation of children's preschool activity as the criterion measure.

\section{Methods}

\section{Subjects}

The aim of the first phase of this study was to calibrate the accelerometer. Twentythree children ages 3 to 5 were recruited to perform five structured activities while wearing single-plane ActiGraph accelerometers (Manufacturing Technology, Inc., Fort Walton Beach, FL). The children's parents provided informed consent prior to data collection, and children or their parents were free to terminate their participation at any time during the testing procedures. Seven children $(30 \%)$ were removed from the sample because of noncompliance with the calibration protocol (e.g., refusal to participate, removal of equipment, completely stopped walking/jogging), leaving a final sample of 16 children. This study was approved by the University of South Carolina Institutional Review Board.

The aim of the second phase of the study was to validate the accelerometer count cutoffs in preschool settings. A total of 281 children were recruited from 9 preschools in the Columbia, SC area. Parents provided informed consent prior to data collection. A final sample of 269 children was retained after deletions for incomplete or missing data. 


\section{Measures}

The ActiGraph is a small $(5.1 \times 3.8 \times 1.5 \mathrm{~cm})$, lightweight $(42.6 \mathrm{~g})$, single plane (vertical) accelerometer that has been validated for use with children in laboratory ${ }^{17}$ and field settings. ${ }^{18,19}$ The ActiGraph collects and stores accelerations between 0.05 Gs and 2.0 Gs. The analog acceleration is converted to a digital signal and this value (count) is stored in user-specified time intervals (epochs). Fifteen-second epochs were used for this study. After data collection, the monitor is downloaded to a computer for subsequent data reduction and analysis.

A modification of the Child Activity Rating Scale (CARS) ${ }^{12}$ was used as the criterion physical activity measure. The CARS is a continuous observation system, designed and validated for use with children age 3 to $4 \mathrm{y}$, which classifies activity into 5 categories. Puhl et al. measured energy expenditure, by indirect calorimetry, in 5 to 6-year-old children performing activities representative of the CARS intensity categories. ${ }^{12}$ Energy expenditure was significantly different $(P<0.05)$ among the five activity intensity categories. Table 1 lists the operational definitions and representative activities used for this study. In the preschool settings, a 15-s momentary time sampling method was used. Each child was observed for $15 \mathrm{~s}$ and then the physical activity and other contextual variables were entered into a handheld computer (Palm, Inc.) for the next $15 \mathrm{~s}$. The time on the handheld computers was synchronized with the computer used to initialize the accelerometers so that observation and ActiGraph data could be temporally matched.

The observation system was developed and pilot tested at a separate preschool during April-September 2000. A videotape of children in the preschool was recorded and the children's activity scored by the project coordinator. This tape was used to calculate intra-observer reliability of four research assistants at the beginning and end of data collection. Intraclass correlation coefficients (ICC) for the physical activity level ranged from 0.95 to 0.96 and 0.88 to 0.94 at baseline and post data collection, respectively. Inter-observer agreement at the mid-point of the study was assessed by simultaneous field observations of the same child, using the project coordinator as the criterion standard. The ICCs ranged from 0.91 to 0.98 . Percent agreement of 15 -s physical activity categorizations across all time points ranged from 75 to $99 \%$ (kappa $=0.66$ to 0.98 ).

For children in both the calibration study and the field trial in the 9 preschools, height was measured to the nearest $0.1 \mathrm{~cm}$ using a portable height

\section{Table 1 Operational Definitions Used for Coding Directly Observed Physical Activity}

\begin{tabular}{|c|c|c|}
\hline Observed activity code & Operational definition & $\begin{array}{l}\text { Representative activity } \\
\text { used for calibration }\end{array}$ \\
\hline 1 & Stationary/motionless & Sitting and talking \\
\hline 2 & $\begin{array}{l}\text { Stationary with movement of limbs } \\
\text { or trunk }\end{array}$ & Sitting and playing \\
\hline 3 & Slow/Easy movement & Slow walking \\
\hline 4 & Moderate movement & Fast walking \\
\hline 5 & Fast movement & Jogging \\
\hline
\end{tabular}


board (Shorr Productions, Olney, MD) and weight to the nearest $0.1 \mathrm{~kg}$ using a calibrated digital scale (model PS6600, BeFour, Inc., Saukville, WI). Body-mass index (BMI) was calculated as the body mass in $\mathrm{kg}$ divided by height in meters squared $\left(\mathrm{kg} / \mathrm{m}^{2}\right)$.

\section{Calibration Procedures}

The ActiGraph accelerometer was calibrated by having children perform five structured activities, based on the CARS intensity categories, for $3 \mathrm{~min}$ each. The activities were completed in the following order: sitting and talking, fast walking, sitting and playing, slow walking, and jogging. All activities were performed in the presence of three research assistants to facilitate compliance with the study protocol. One of the research assistants performed each activity with the child and paced them during the walking and jogging conditions. The average walking and running speeds $(\mathrm{km} / \mathrm{hr})$ were, $3.2 \pm 0.6,4.3 \pm 0.6$, and $6.9 \pm 3.9$ for the slow walking, fast walking, and jogging conditions, respectively. Most children stopped walking or running for several seconds at some point during the protocol. None of the children included in the final sample stopped performing a task for more than $15 \mathrm{~s}$. While the included participants were not perfectly obedient, their slight deviations from the protocol did not warrant their removal. Having several research assistants present, all providing verbal encouragement and one performing the activities with the child, helped prevent further attrition. To assess inter-instrument reliability, children wore an ActiGraph on each hip, anterior to the iliac crest, using an adjustable elastic belt. A digital watch was synchronized daily with the computer used to initialize the activity monitors, and this watch was used to record the start and stop times for each of the activities. These times were used to extract the corresponding activity monitor data.

The average ActiGraph counts per activity were calculated separately for the left and right hip monitors using all 3 min of each activity. Inter-instrument reliability for the monitors was evaluated by calculating the intraclass correlation coefficient (ICC) between the left and right hip monitors. A two-way analysis of variance (ANOVA) was also calculated to compare monitor counts from left and right hip monitors for the five structured activities. The ICC between left and right hip monitors was $r=0.84$. ANOVA revealed that the hip main effect $(N=$ $17, F=0.06, P=0.80)$ and the activity by hip interaction $(N=17, F=0.07, P=$ $1.00)$ were both nonsignificant. Therefore, only data from the right hip monitor was used for the rest of the analysis and only one monitor was used in the field settings.

\section{Evaluation Procedures}

To evaluate the ability of the count cutoffs to categorize activity intensity, children wore one ActiGraph on their right hip during the entire time they were at their preschool for up to 10 consecutive weekdays. As part of a larger study exploring associations between physical activity and the preschool environment, a research assistant observed and recorded the child's activity during one to three 1-h sessions on separate days over the same 10-d period. Observations were scheduled to avoid lunch and nap times. Data collection was conducted from October 2000 to May 2001. 


\section{Data Reduction and Analysis}

SAS version 8.02 software was used for all statistical analyses. Significance was set at the $P=0.05$ level. One-way ANOVAs were calculated to detect differences in ActiGraph average 15-s count values among the five activities. Wearing the ActiGraph on the hip does not allow for the detection of limb movement. Therefore, both sitting activities (observation codes 1 and 2) were combined to produce one sedentary intensity category. Slow walking, fast walking, and jogging were categorized as light, moderate, and vigorous intensity, respectively.

Receiver operating characteristic (ROC) curves were calculated using SAS Proc Logistic to determine age-specific ActiGraph count cutoff values for the four intensity categories. ROC curve analysis is derived from clinical diagnostic tests to differentiate, for example, between normal and diseased states. Using a given cutoff to distinguish the diseased state, a certain percentage of cases will be true positives, true negatives, false positives, and false negatives. By identifying a range of possible cutoffs, the cutoff at which the rate of true positives (sensitivity) and true negatives (specificity) are maximized can then be determined. The ROC curve is plotted with sensitivity along the $y$-axis and 1 -specificity on the $x$-axis. ${ }^{20}$

The area under the ROC curve is interpreted as a measure of the accuracy of a clinical test to discriminate between 2 populations. For example, an area of 0.84 means that a randomly selected individual from the positive group has a test value larger than that for a randomly chosen individual from the negative group $84 \%$ of the time. ${ }^{21}$ When the variable under study cannot distinguish between the 2 groups (i.e., where there is no difference between the 2 distributions) the area will be equal to 0.5 (the ROC curve will coincide with the diagonal). When there is a perfect separation of the values of the 2 groups (i.e., there is no overlapping of the distributions), the area under the ROC curve equals 1 (the ROC curve will reach the upper left corner of the plot). The $95 \%$ confidence interval for the area can be used to test the hypothesis that the theoretical area is 0.5 . If the confidence interval does not include the 0.5 value, then there is evidence that the laboratory test does have the ability to distinguish between the 2 groups. ${ }^{20,21}$

Age-specific ActiGraph count cutoffs were calculated based on previous research indicating an age-related increase in count cutoffs for children in 1st through 12th grade. ${ }^{22}$ Thus, the ROC curve analyses were calculated separately for the 3, 4, and 5 y olds. For each age group, the intensity-specific ROC curves were calculated by dummy coding the calibration activities ( 0 and 1$)$ and using this variable as the dependent variable in the logistic regression model. The independent variable was the subject's average 15-s count values for each calibration activity. For sedentary intensity, activities were coded as either sedentary (1; both sitting activities) or nonsedentary (0; walking and jogging activities). Similarly, to calculate an ROC curve for moderate-to-vigorous physical activity (MVPA), activities were coded as less than moderate intensity $(0$; sitting and slow walking activities) and moderate or greater intensity activities (1; fast walking and jogging). Vigorous intensity was identified by dichotomizing the activities into vigorous (1; jogging) and less than vigorous activities ( 0 ; sitting and walking activities). The count cutoffs were then selected from the point on the age- and intensity-specific ROC curve that maximized both sensitivity and specificity. The sedentary cutoff is defined as sedentary (sitting) or not (at least light intensity activity). Similarly, MVPA is defined as moderate (at least moderate intensity activity) or not (light 
and sedentary intensity activity). Therefore, these 2 cutoffs provide the boundary for the light intensity cutoffs and it was not necessary to calculate ROC curves for the light intensity category.

As a result of the observation system's time-sampling procedure, physical activity was observed during every other 15-s interval for the 1-h observations conducted in the preschool settings. Therefore, only the ActiGraph data that corresponded to the observed intervals were used for the correlation analysis so that accelerometer and observation data were matched for each observation hour. Observation data were reduced to a total physical activity score by summing the observed 15-s activity scores for each observation hour. The total number of observed 15-s intervals classified as sedentary (SED), light (LIG), moderate (MOD), moderateand-vigorous physical activity (MVPA), and vigorous (VIG) were also calculated. Analogous variables were calculated from the ActiGraph data using custom software. These variables included the total ActiGraph counts over the observation hour and the number of time intervals spent in the various activity intensities using the calibration count cutoffs. Pearson correlations were calculated between analogous ActiGraph and observation variables using log-transformed values for variables with skewed distributions.

\section{Results}

Characteristics of the children who participated in the calibration study and the field evaluation are provided in Table 2. The relatively high mean BMI for the $3 \mathrm{y}$-olds in the calibration sample is the result of one overweight subject (BMI $=24.5$ ). Without this child, the BMI for the 3 y-olds was 13.9. This subject was retained for analysis because removing him did not alter the results. The majority

Table 2 Characteristics of Calibration and Field Evaluation Samples

\begin{tabular}{lccc}
\hline & $3 \mathrm{y}$ & $4 \mathrm{y}$ & $5 \mathrm{y}$ \\
\hline Calibration & & & \\
$N$ & 5 & 5 & 6 \\
Male $(\%)$ & 60.0 & 50.0 & 83.3 \\
Race $(\%$ white $)$ & 100.0 & 50.0 & 33.3 \\
Height $(\mathrm{cm})^{\mathrm{a}}$ & $105(3.0)$ & $108(5.5)$ & $118(5.0)$ \\
Weight $(\mathrm{kg})^{\mathrm{a}}$ & $17.9(5.40)$ & $17.9(2.53)$ & $24.4(4.12)$ \\
BMI $\left(\mathrm{kg} / \mathrm{m}^{2}\right)$ & $16.1(4.76)$ & $15.4(1.33)$ & $17.5(2.05)$ \\
Field evaluation & & & \\
$N$ & 69 & 125 & 75 \\
Male $(\%)$ & 42.0 & 48.0 & 48.0 \\
Race $(\% \mathrm{white})$ & 37.7 & 25.6 & 38.7 \\
Height $(\mathrm{cm})^{\mathrm{b}}$ & $104(4.9)$ & $108(5.5)$ & $116(4.9)$ \\
Weight $(\mathrm{kg})^{\mathrm{b}}$ & $16.7(2.63)$ & $19.0(3.22)$ & $22.1(3.80)$ \\
BMI $\left(\mathrm{kg} / \mathrm{m}^{2}\right)^{\mathrm{c}}$ & $15.4(1.41)$ & $16.1(1.74)$ & $16.5(1.83)$ \\
\hline
\end{tabular}

Values are percent or means ( \pm standard deviation). ${ }^{5} 5$ y-olds significantly different from 3 - and 4-y-olds; ${ }^{b}$ all ages significantly different from each other; ${ }^{c} 3$-y-olds significantly different from 4- and 5-y-olds. 
of children in the field evaluation were age 4, approximately half of the sample was male, and $61 \%$ was African American.

The differences in ActiGraph counts by calibration activity level are shown in Figure 1. Similar results were obtained using average heart rates obtained over the last 2 min of each calibration activity (not shown). ActiGraph counts were significantly different among all activities except between the two sitting activities. This was not surprising because there was little to no vertical displacement during these activities, and the accelerometer would not be able to distinguish between such movements.

The age-specific 15-s count cutoffs, sensitivity, specificity, and area under the ROC curve are provided in Table 3. For light activity, sensitivity, specificity, and area under the curve were not calculated from the ROC curve analysis. Thus, the ActiGraph count ranges for light intensity were calculated as the range of values between the sedentary and MVPA count cutoffs. Sensitivity and specificity ranged from $86.7 \%$ to $100.0 \%$ and $66.7 \%$ to $100.0 \%$, respectively. Each curve represented excellent discrimination as determined by area under the ROC curve $(>0.90)$, and the confidence intervals did not include 0.5. An example of these curves is provided in Figure 2 for the MVPA count cutoff for 4 y-olds. Each data point represents a 15-s count value recorded by the ActiGraph during the calibration procedures. The 25 data points are from five $4 \mathrm{y}$-old subjects with 1 data point for each of the 5 calibration activities. The lowest ( 8 counts/15 s) and highest count values (1597 counts/15 s) recorded during the sitting and jogging activities, respectively, are

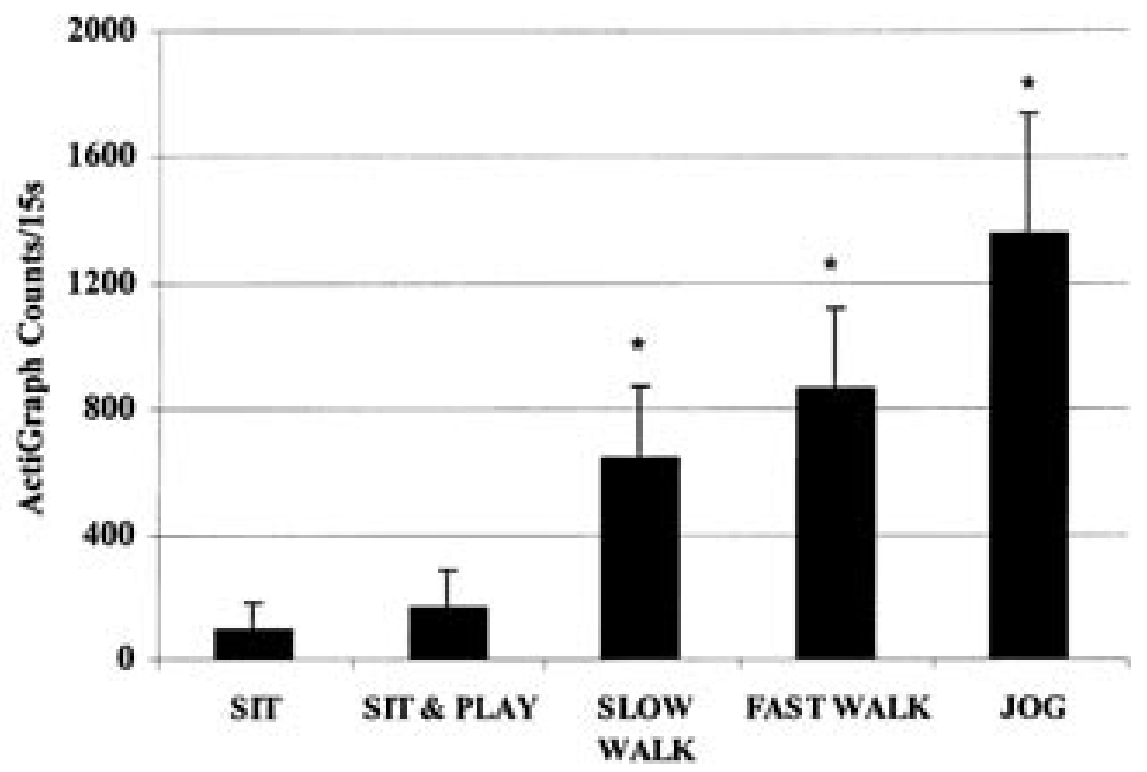

\footnotetext{
Activity

Figure 1 - ActiGraph monitor counts by activity (mean + standard deviation); *significant difference in ActiGraph counts between activities.
} 
Table 3 Sensitivity, Specificity, Area Under the ROC Curve, and 15-s ActiGraph Count Cutoffs Based on Age-Dependent ROC Curves

\begin{tabular}{|c|c|c|c|c|c|}
\hline Activity/age & $\begin{array}{l}\text { Intensity } \\
\text { category }\end{array}$ & $\begin{array}{l}\text { Sensitivity } \\
(\%)\end{array}$ & $\begin{array}{l}\text { Specificity } \\
(\%)\end{array}$ & $\begin{array}{l}\text { Area under ROC } \\
\text { curve }(95 \% \mathrm{CI})\end{array}$ & $\begin{array}{l}\text { Counts/ } \\
15 \mathrm{~s}\end{array}$ \\
\hline $\begin{array}{l}\text { Sit and sit } \\
\& \text { play }\end{array}$ & Sedentary & & & & \\
\hline 3 y old & & 100.0 & 100.0 & $1.00(1.00-1.00)$ & $0-301$ \\
\hline 4 y old & & 100.0 & 100.0 & $1.00(1.00-1.00)$ & $0-363$ \\
\hline 5 y old & & 94.4 & 91.7 & $0.97(0.91-1.00)$ & $0-398$ \\
\hline Slow walk & Light & & & & \\
\hline 3 y old & & N/A & N/A & N/A & $302-614$ \\
\hline 4 y old & & N/A & N/A & N/A & $364-811$ \\
\hline 5 y old & & N/A & N/A & N/A & $399-890$ \\
\hline Fast walk & Moderate & & & & \\
\hline 3 y old & & 93.3 & 100.0 & $0.97(0.92-1.00)$ & $615-1230$ \\
\hline 4 y old & & 86.7 & 90.0 & $0.95(0.88-1.00)$ & $812-1234$ \\
\hline 5 y old & & 94.4 & 66.7 & $0.92(0.83-1.00)$ & $891-1254$ \\
\hline Jog & Vigorous & & & & \\
\hline 3 y old & & 100.0 & 80.0 & $0.96(0.88-1.00)$ & $\geq 1231$ \\
\hline 4 y old & & 100.0 & 80.0 & $0.96(0.88-1.00)$ & $\geq 1235$ \\
\hline 5 y old & & 95.8 & 83.3 & $0.97(0.90-1.00)$ & $\geq 1255$ \\
\hline
\end{tabular}

Note. N/A, not calculated using ROC curve analysis.

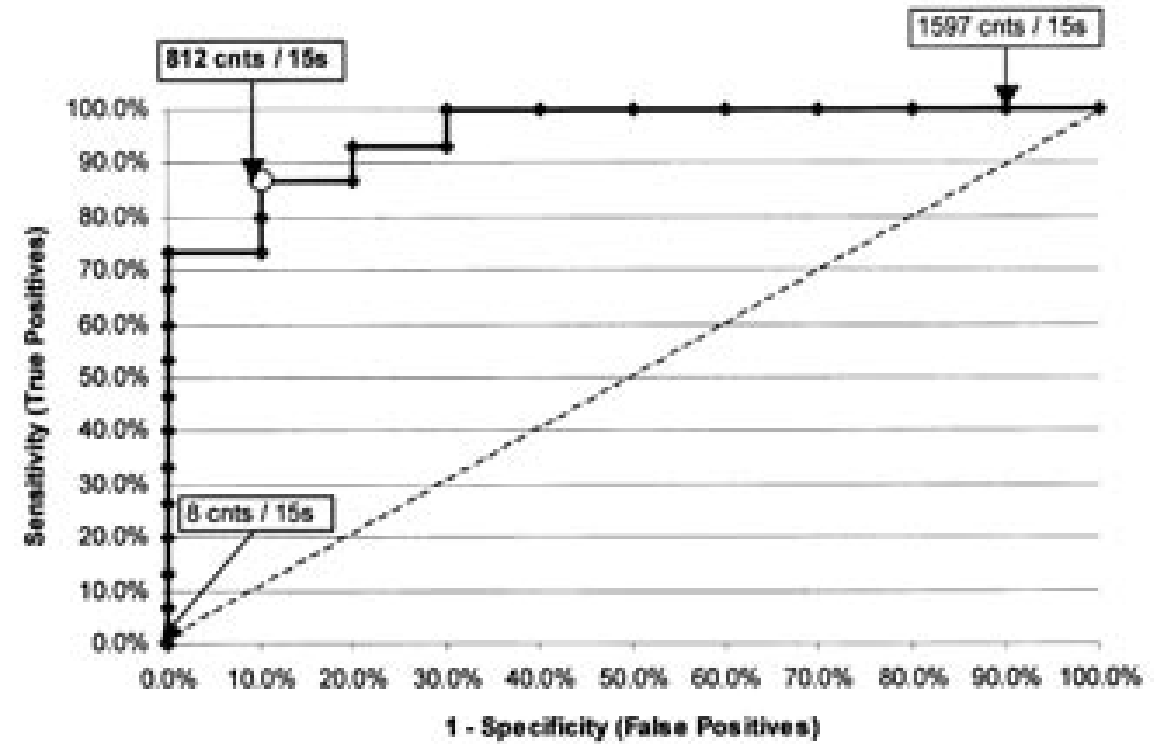

Figure 2 - Sample ROC curve of MVPA for 4-y-olds. $\bigcirc$ Associated with an ActiGraph count value of 812 counts/15-s at $86.7 \%$ sensitivity, $90 \%$ specificity. Approximate area under the curve $=0.95 . \ldots \ldots$. $\ldots$ no discrimination. 
also indicated on the ROC curve. Labels for all data points are not shown. The dashed line intersecting $(0,0)$ and $(100,100)$ indicates no discrimination between an event versus nonevent (e.g., MVPA vs. non-MVPA). The data point at $(86.7 \%$ and $90.0 \%$ ) represents a 15 -s count value of 812 and was used as the point that provided the highest sensitivity and specificity. This determination is somewhat subjective given that the data point at $(93.3 \%$ and $80.0 \%)$ is comparable but there is a trade-off between the sensitivity and specificity. Using 812 counts per $15 \mathrm{~s}$ as the $4 \mathrm{y}$-old MVPA cutoff is slightly more conservative since the false positive rate (1- specificity) is slightly lower (10\% vs. $20 \%)$.

Pearson correlation coefficients between direct observation physical activity variables and ActiGraph variables collected during the field trial in the 9 preschools are presented in Table 4 . Correlations are moderate in magnitude $(0.46$ to 0.70$)$ and all are statistically significant $(P<0.001)$.

Table 4 Pearson Correlation Coefficients Between Direct Observation Scores and ActiGraph Variables

\begin{tabular}{|c|c|c|c|c|c|c|}
\hline \multirow[b]{2}{*}{ Observation } & \multicolumn{6}{|c|}{ Log-transformed ActiGraph variables } \\
\hline & $\begin{array}{l}\text { Total } \\
\text { counts }\end{array}$ & $\begin{array}{l}\text { SED } \\
\text { epochs }\end{array}$ & $\begin{array}{l}\text { LIG } \\
\text { epochs }\end{array}$ & $\begin{array}{l}\text { MOD } \\
\text { epochs }\end{array}$ & $\begin{array}{l}\text { MVPA } \\
\text { epochs }\end{array}$ & $\begin{array}{l}\text { VIG } \\
\text { epochs }\end{array}$ \\
\hline Log total score & $0.58 *$ & & & & & \\
\hline SED epochs & & $0.70^{*}$ & & & & \\
\hline LIG epochs & & & $0.59 *$ & & & \\
\hline Log MOD epochs & & & & $0.50 *$ & & \\
\hline Log MVPA epochs & & & & & $0.46^{*}$ & \\
\hline Log VIG epochs & & & & & & $0.61 *$ \\
\hline
\end{tabular}

${ }^{*} P<0.001$; SED, sedentary; LIG, light; MOD, moderate; MVPA, moderate-to-vigorous physical activity; VIG, vigorous; epochs, data collection time interval (15-s).

\section{Discussion}

This study established age-specific count cutoffs for the ActiGraph accelerometer when used to evaluate physical activity in 3 to 5 y-old children. The values are specific to data collected in 15-s time intervals. This is the first study to establish separate cutoffs representing sedentary, light, moderate-to-vigorous, and vigorous physical activity in 3, 4, and 5 y-old children.

Two previous studies identified accelerometer cutoffs in preschool children, but they only determined differences between sedentary and nonsedentary activity. ${ }^{14,16}$ Using the CARS direct observation system as the criterion measure, but a different accelerometer (Actiwatch model W16, Mini Mitter Co., Bend, OR), Finn et al. found that a cutpoint of $>1000$ counts/min represented nonsedentary activity; ${ }^{14}$ however, direct comparisons with the present study are difficult because of the use of different accelerometers. Reilly et al. validated the ActiGraph accelerometer using the Children's Physical Activity Form (CPAF) observation system as the criterion measure. ${ }^{16}$ Using ROC curve analysis, they determined that $>1100$ counts $/ \mathrm{min}$ provided the optimal sensitivity $(83 \%)$ and specificity $(82 \%)$ for identifying minutes 
of nonsedentary activity. This sedentary intensity cutoff is slightly lower than those found in this study, if multiplied by 4 to approximate a 1-min count cutoff (e.g., 300 counts $/ 15 \mathrm{sec} \times 4=1200$ counts $/ \mathrm{min}$ for $3 \mathrm{y}$-olds). (It is assumed that multiplying 15 -s values by 4 approximates the 1-min count values, although it remains unclear if this is an appropriate comparison.) The difference in these sedentary cutoffs might be caused by differences in study design. The present study used structured activities that fit the CARS activity category descriptions. In contrast, Reilly et al. used unstructured activities that fit the CPAF observation system.

The age-specific count cutoffs for 3, 4, and $5 \mathrm{y}$-olds are a unique element of the present study. Using inappropriate cutoffs could reduce the validity of physical activity categorizations for some age groups. Previous research has shown that using one cutoff for all children would reduce the likelihood that younger children would meet the cutoff and result in those children appearing to be less active. ${ }^{22}$ Others have used one cutoff for all subjects in the 3 to 5 y age range, ${ }^{14,16}$ but the age-specific cutoffs identified in the current study suggest that a single cutoff for this age group might not be adequate.

It is unknown whether age itself or other unknown moderating factors, such as maturational age, changes in vertical displacement, and overall efficiency during locomotion, could be responsible for the differences. The use of age-specific count cutoffs was based on 1) previous research using regression analyses to identify count cutoffs for school age children, ${ }^{22}$ and 2) the ease of obtaining age compared to other physical or physiological measures. Variables such as gender and race might affect levels of physical activity (e.g., girls and minority children being less active than boys and Caucasians), but could have limited applicability to the calibration and validation of an accelerometer. The issue is whether the variable moderates the vertical accelerations recorded by the ActiGraph during physical activities. Until puberty, gender likely has little effect on how an individual moves and there seems no biological plausibility to suspect that race might moderate the accelerations detected by a vertical plane accelerometer. One could hypothesize, however, that a child with a greater BMI might have blunted vertical displacement during locomotion because of the extra work required to move their bodies. Height, leg length, coordination, and maturational age could all affect the vertical accelerations detected by the accelerometer, independent of chronological age. The impact of these physical characteristics, and possibly others, on the accelerometer output needs further investigation but in larger samples than is available in this study.

Fifteen-second time intervals were used in collecting the accelerometer data for this study. The use of accelerometer epochs of less than $1 \mathrm{~min}$ has been proposed as a means of more accurately describing the activity intensity patterns of children owing to the intermittent nature of children's physical activity. ${ }^{23-25}$ Therefore, using 1-min intervals to record young children's physical activity via accelerometry could mask their true physical activity level. For example, an observer might witness a short bout (10 s) of nonsedentary activity during a given 1-min interval, but this level of physical activity might not meet the accelerometer cutoff as it was averaged with $50 \mathrm{~s}$ of sedentary counts during that same 1-min interval. This could have resulted in the lower sedentary cutoff in the Reilly et al. study, since they used 1-min intervals in field settings rather than steady state activity like the structured activities performed by the children in the current study. In such a validation study, the mean observed activity level recorded by the observers (using the CPAF protocol) would be associated with a slightly lower count value from the accelerometer. 
Receiver Operating Characteristic curve analysis was used to determine the ActiGraph intensity cutoffs for sedentary, moderate, and vigorous physical activity. This method was used because the dependent variable, derived from the direct observation system, was a nominal level variable with only 4 categories. The Receiver Operating Characteristic curve technique is especially well suited for establishing cut-points as it is based on maximizing specificity and sensitivity. Regression techniques that require a continuous or nearly continuous dependent variable did not fit the current data set. If the dependent variable had been a continuous measure (e.g., energy expenditure measured in $\mathrm{mL} \cdot \mathrm{kg} \cdot \mathrm{min}^{-1}$ ) then the regression approach would have been a more appropriate analytic tool. The high area under the curve for each age- and intensity-specific curve $(>0.90)$ indicates that the cutoffs determined from these curves provide excellent discrimination among the activity intensity categories.

In the field tests in the 9 preschools, the ActiGraph was able to discriminate between the structured sedentary, light, moderate, and vigorous intensity physical activities of 3 to 5 y-old children. The time spent in these activity intensity categories, using the 15-s, age-specific count cutoffs, was significantly associated with directly observed time spent in these categories in the field settings. These results are similar to the findings of Reilly et al. in which accelerometer counts per minute differed significantly between CPAF categories.$^{16}$ Being able to identify the amount of time spent in a range of intensity categories is useful, since physical activity recommendations for children specify 60 -min of moderate-to-vigorous intensity activity on all days. ${ }^{26,27}$

The present study found correlations between ActiGraph counts and observation scores of 0.46 to 0.70 . Several previous studies have attempted to validate accelerometers with preschool-age children. ${ }^{13,15,28}$ Our correlations are slightly lower than those reported by Fairweather et al. ( $r=0.79$ to 0.87$)$. They measured total activity using a previous model of the ActiGraph and a different observational system (CPAF), however, and did not distinguish among various activity intensities. ${ }^{13}$ Finn et al. found correlations of 0.03 to 0.92 (median, 0.74) in a study that used a different accelerometer but the same observation system (CARS) as the current study. Although they used different instrumentation, the Fairweather study, the Finn study, and the present study all concluded that the accelerometer is an appropriate tool for assessing physical activity in preschool children.

This study is limited by the small convenience sample used for the calibration of the ActiGraph. The significant associations between ActiGraph data and direct observation scores from the large field sample and the high area under the ROC curves, however, indicate that the count cutoffs were able to discriminate between the activity intensities. In the field settings, observers were instructed to identify the highest level of activity that occurred during each 15-s observation interval. In contrast, the ActiGraph records the total accelerations accumulated during the 15-s interval. These 2 approaches, while related, are measuring slightly different aspects of the child's activity and might have attenuated associations between these methods because of children's intermittent activity patterns. For example, observers might have recoded an observation interval as vigorous for a short burst of movement while the ActiGraph might record the same interval at a lower intensity level given that the total counts for that 15 -s interval might not have been enough to reach the vigorous intensity cutoff. While this discrepancy was not an issue during calibration, because the activity was structured and the intensity remained consistent for 
each 3-min segment, the associations between the direct observation scores and ActiGraph output in the field settings were likely attenuated by this methodological difference. Lastly, using a measure of energy expenditure $\left(\mathrm{mL} \cdot \mathrm{kg} \cdot \mathrm{min}^{-1}\right)$, instead of the observation categories, would provide a continuous, objective, and physiologically meaningful dependent variable with which to compare both the observation and ActiGraph data against. The use of indirect calorimetry is difficult in this young population, however, because of poor subject compliance, and the activities could be performed differently because of equipment constraints and at a greater metabolic cost resulting from increased physical or emotional stress.

\section{Conclusion}

In conclusion, the ActiGraph count cutoffs established in this study can be used to categorize the time that preschool-age children spend in different physical activity intensity levels. This methodology allows for an objective and feasible alternative to subjective parent or teacher proxy reports and provides a valid measurement tool for cross-sectional and experimental research investigating young children's physical activity levels.

\section{Acknowledgments}

This research was supported by a grant from Gerber Products, Inc. The authors thank the administration, staff, parents, and students at all of the participating preschools in the Columbia, SC area. We also thank Gaye Groover Christmus, MPH, for editorial assistance in the preparation of the manuscript.

\section{References}

1. Ogden CL, Flegal KM, Carroll MD, Johnson CL. Prevalence and trends in overweight among US children and adolescents, 1999-2000. JAMA. 2002;288(14):1728-1732.

2. de OM, Blossner M. Prevalence and trends of overweight among preschool children in developing countries. Am J Clin Nutr. 2000;72(4):1032-1039.

3. Martorell R, Kettel KL, Hughes ML, Grummer-Strawn LM. Overweight and obesity in preschool children from developing countries. Int $J$ Obes Relat Metab Disord. 2000;24(8):959-967.

4. Crespo CJ, Smit E, Troiano RP, Bartlett SJ, Macera CA, Andersen RE. Television watching, energy intake, and obesity in US children. Results from the Third National Health and Nutrition Examination Survey, 1988-1994. Arch Pediatr Adolesc Med. 2001;155(3):360-365.

5. Dietz WH, Gortmaker SL. Preventing obesity in children and adolescents. Ann Rev Public Health. 2001;22:337-353.

6. Fulton JE, Burgeson CR, Perry GR, et al. Assessment of physical activity and sedentary behavior in preschool-age children: priorities for research. Pediatr Exerc Sci. 2001;12: 113-126.

7. Sallis JF. Self-report measures of children's physical activity. J Sch Health. 1991;61(5): 215-219.

8. Halverson Jr CF, Waldrop MF. The relations of mechanically recorded activity level to varieties of preschool play behavior. Child Develop. 1973;44:678-681. 
9. Manios Y, Kafatos A, Markakis G. Physical activity of 6-year-old children: validation of two proxy reports. Pediatr Exerc Sci. 1998;10(2):176-188.

10. Noland M, Danner F, DeWalt K, McFadden M, Kotchen JM. The measurement of physical activity in young children. Res Q Exerc Sport. 1990;61(2):146-153.

11. Sirard J, Pate RR. Physical activity assessment in children and adolescents. Sports Med. 2001;31:439-454.

12. Puhl J, Greaves K, Hoyt M, Baranowski T. Children's Activity Rating Scale (CARS): description and calibration. Res Q Exerc Sport. 1990;61(1):26-36.

13. Fairweather SC, Reilly JJ, Grant S, Whittaker A, Paton JY. Using the Computer Science and Applications (CSA) activity monitor in preschool children. Pediatr Exerc Sci. 1999;11:413-420.

14. Finn K, Johannsen N, Specker B. Factors associated with physical activity in preschool children. J Pediatr. 2002;140(1):81-85.

15. Mukeshi M, Gutin B, Anderson WA, et al. Validation of the Caltrac movement sensor using direct observation in young children. Pediatr Exerc Sci. 1990;2(3):249-254.

16. Reilly JJ, Coyle J, Kelly L, Burke G, Grant S, Paton JY. An objective method for measurement of sedentary behavior in 3- to 4-year olds. Obes Res. 2003;11:1155-1158.

17. Trost SG, Ward DS, Moorehead SM, Watson PD, Riner W, Burke JR. Validity of the Computer Science and Applications (CSA) activity monitor in children. Med Sci Sports Exerc. 1998;30(4):629-633.

18. Ott AE, Pate RR, Trost SG, Ward DS, Saunders R. The use of uniaxial and triaxial accelerometers to measure children's "free-play" physical activity. Pediatr Exerc Sci. 2000;12(4):360-370.

19. Eston RG, Rowlands AV, Ingledew DK. Validity of heart rate, pedometry, and accelerometry for predicting the energy cost of children's activities. J Appl Physiol. 1998;84(1):362-371.

20. Hanley JA, McNeil BJ. The meaning and use of the area under a receiver operating characteristic (ROC) curve. Radiology. 1982;143(1):29-36.

21. Zweig MH, Campbell G. Receiver-operating characteristic (ROC) plots: a fundamental evaluation tool in clinical medicine. Clin Chem. 1993;39(4):561-577.

22. Freedson PS, Sirard J, Debold E, et al. Calibration of the Computer Science and Applications, Inc. (CSA) accelerometer. Med Sci Sports Exerc. 1997;29:S45.

23. Nilsson A, Ekelund L, Yngve A, Sjostrom M. Assessing physical activity among children using different time sampling intervals and placements. Pediatr Exerc Sci. 2002;14(1):87-96.

24. Welk GJ, Corbin CB, Dale D. Measurement issues in the assessment of physical activity in children. Res Q Exerc Sport. 2000;71(2 Suppl):S59-S73.

25. Bailey RC, Olson J, Pepper SL, Porszasz J, Barstow TJ, Cooper DM. The level and tempo of children's physical activities: an observational study. Med Sci Sports Exerc. 1995;27(7):1033-1041.

26. Biddle S, Sallis JF, Cavill NA. Young and active? Young people and health enhancing physical activity. Evidence and implication. London: Health Education Authority; 1998.

27. National Association for Sport and Physical Education. Active Start: A Statement of Physical Activity Guidelines for Children Birth to Five Years. Reston, VA: NASPE Publications; 2002.

28. Finn KJ, Specker B. Comparison of Actiwatch activity monitor and Children's Activity Rating Scale in children. Med Sci Sports Exerc. 2000;32:1794-1797. 